UCT-TP-282/10

$\mathrm{MZ}-\mathrm{TH} / 10-40$

October 13, 2018

\title{
Confronting electron-positron annihilation into hadrons with QCD: an operator product expansion analysis 1
}

\author{
S. Bodenstein ${ }^{(a)}$, C. A. Dominguez ${ }^{(a),(b)}$, S. I. Eidelman ${ }^{(c),(d)}$, \\ H. Spiesberger ${ }^{(e)}$, K. Schilcher ${ }^{(e)}$ \\ ${ }^{(a)}$ Centre for Theoretical \& Mathematical Physics, \\ University of Cape Town, Rondebosch 7700, South Africa \\ ${ }^{(b)}$ Department of Physics, Stellenbosch University, Stellenbosch 7600, South Africa \\ (c) Budker Institute of Nuclear Physics, 630090, Novosibirsk, Russian Academy of \\ Science, Russia \\ (d) Novosibirsk State University, 630090, Novosibirsk, Russia \\ (e) Institut für Physik, Johannes Gutenberg-Universität Staudingerweg 7, D-55099 \\ Mainz, Germany
}

\begin{abstract}
Experimental data on the total cross section of $e^{+} e^{-}$annihilation into hadrons are confronted with QCD and the operator product expansion using finite energy sum rules. Specifically, the power corrections in the operator product expansion, i.e. the vacuum condensates, of dimension $d=2,4$ and 6 are determined using recent isospin $I=0+1$ data sets. Reasonably stable results are obtained which are compatible within errors with values from $\tau$-decay. However, the rather large data uncertainties, together with the current value of the strong coupling constant, lead to very large errors in the condensates. It also appears that the separation into isovector and isoscalar pieces introduces additional uncertainties and errors. In contrast, the high precision $\tau$-decay data of the ALEPH collaboration in the vector channel allows for a more precise determination of the condensates. This is in spite of QCD asymptotics not quite been reached at the end of the $\tau$ spectrum. We point out that isospin violation is negligible in the integrated cross sections, unlike the case of individual channels.
\end{abstract}

\footnotetext{
${ }^{1}$ Supported in part by the National Research Foundation (South Africa), and the Alexander von Humboldt Foundation (Germany).
} 


\section{Introduction}

In the framework of QCD sum rules [1]-[2], the quark and gluon propagator modifications due to confinement are parametrized in terms of vacuum expectation values of quark and gluon fields. These so-called vacuum condensates appear as power corrections to perturbative QCD in the operator product expansion (OPE) of current correlators at short distances. The QCD condensates are accessible from experiment through the spectral functions, i.e. the imaginary parts of the current correlators. Since from dimensional arguments there are no gauge invariant operators of dimension $d=2$ built from quark and/or gluon fields in QCD, it has been customary to assume these power corrections to start at $d=4$. However, one cannot exclude a priori some dynamical mechanism capable of generating a term of $d=2$ in the OPE [3]. In a recent paper [4] it has been argued that there is no evidence for such a term in lattice QCD, contrary to earlier claims. There is also no definite evidence from $\tau$-decay as shown in recent sum rule analyses [5]-[8] based on the final ALEPH data [9]. These determinations have also found values for the dimension $d=4$ and $d=6$ condensates consistent with expectations.

Given the impact of these condensates on QCD sum rule applications it is important to attempt a determination based on experimental information independent of $\tau$-decay. Historically, determinations based on $e^{+} e^{-}$annihilation data have preceded $\tau$-decay analyses [10. Given the current availability of more accurate data from $e^{+} e^{-}$annihilation, and the sizable increase in the value of the strong coupling over the years, it is imperative to perform a fully updated determination of the condensates to confront with results from $\tau$-decay. An additional motivation for such a project is related to the current discrepancy in the hadronic vacuum polarization value of the $g-2$ factor of the muon obtained from $\tau$-decay and from $e^{+} e^{-}$annihilation data [11, to wit. The Standard Model (SM) predictions of $a_{\mu}$, the anomalous magnetic moment of the muon, are limited in precision by contributions from the hadronic vacuum polarization. The dominant terms are usually calculated via a weighted dispersion relation involving experimental cross section data on $e^{+} e^{-}$annihilation into hadrons at low energies, and perturbative QCD (PQCD) at high energies. The required integration kernel emphasizes low photon virtualities. During the last decade the SM prediction of $a_{\mu}$ has improved continuously as more precise experimental data became available [12]. Nonetheless, a discrepancy between the measured and the predicted values remains and it is tempting to trace this discrepancy back to new physics beyond the SM. Unfortunately, the experimental hadronic data entering the analysis originate from different experimental groups and often errors do not overlap. In addition, most of the modern experiments are dominated by systematic uncertainties which are difficult to estimate. Consequently, one should test the data base to the greatest possible extent against the underlying theory, i.e. QCD, before accepting the drastic conclusion that the SM has failed to describe the data.

In the present paper we attempt to investigate the status of experimental $e^{+} e^{-}$annihilation data by confronting them with the OPE using QCD sum rules. We study the first three moments $M_{N}\left(s_{0}\right)(N=0,1,2)$ of $R(s)$, the ratio of the cross sections for hadron to muon production in $e^{+} e^{-}$annihilation, at a finite upper limit $s_{0}$ of the integra- 
tion over the square of the center-of-mass energy, $s_{0}$. These moments are related to the QCD condensates of dimension $d=2 N+2$. The extracted values of these condensates are very sensitive to experimental and theoretical errors as they result from evaluating a small difference between two large numbers, i.e. the difference between integrated data and PQCD. The sum rules therefore constitute a sensitive test of the consistency of the data with general expectations from QCD. In parallel with the determination of a potential $d=2$ term in the OPE, we also consider power corrections of dimension $d=4$ and $d=6$. This can help establish whether there is evidence for a dynamical $d=2$ power correction, and whether the results for the higher dimensional condensates are consistent with expectations. There is also independent information on the $d=4$ gluon condensate from charmonium analyses, as well as theoretical estimates of the $d=6$ four-quark condensate which can be used to check for consistency. We limit the analysis to dimension $d=6$, as higher dimensional condensates are affected by such large uncertainties that no meaningful value can be obtained at present.

In the evaluation of the moments, the upper limit of the sum rule integral must be taken large enough for PQCD to be applicable. It should be recalled in this connection that the QCD asymptotic regime is reached in the time-like region only at energies much higher than in the space-like region. For instance, this is seen in $\tau$-decay data of the ALEPH collaboration [9], where the $V-A$ hadronic spectral function does not agree with the vanishing result of PQCD even at $s_{0} \approx m_{\tau}^{2}$. In contrast, deep inelastic electron-nucleon scattering is well known to exhibit precocious scaling.

In connection with the determination of the muon magnetic moment anomaly, $a_{\mu}$, only the sum of the isospin $I=1$ and $I=0$ spectral functions is needed. However, QCD also makes definite predictions for the moments of the separate isovector and isoscalar components of the spectral functions. The corresponding moments are related by a trivial overall factor determined by the quark charges. Therefore, from the theoretical point of view it is interesting to study both the isovector and the isoscalar channels of $R(s)$ separately. Of specific interest is the $I=1$ channel since independent data are available in this case from hadronic $\tau$-decay. For example, it was suggested in [13] to use the $\tau$ spectral functions in the calculation of the muon anomaly. To be precise, the authors of [13. replaced the $\tau \rightarrow \nu_{\tau}+\pi^{+} \pi^{0}, 2 \pi^{-} \pi^{+} \pi^{0}$ and $\pi^{-} 3 \pi^{0}$ spectral functions measured in $\tau$ decay with the poorly known neutral $2 \pi$ and $4 \pi$ spectral functions from $e^{+} e^{-}$annihilation. For these exclusive final states, sizable model-dependent isospin-breaking effects need to be incorporated. This is in contrast to the case of moments, as opposed to individual channels, where isospin breaking is negligible for large enough $s_{0}$. But if the aim is to determine QCD condensates for definite isospin, then the necessary separation of $e^{+} e^{-}$ data into final states with $I=0$ or $I=1$ would require a detailed understanding of isospin violation. This separation, though, would not even be possible in the presence of mixing. This mixing has been known for quite some time to be sizable in the $\rho-\omega$ system. In addition, it was shown recently that $\rho-\gamma$ mixing could, perhaps, explain the well-known discrepancy between $e^{+} e^{-}$- and $\tau$-decay-based evaluations of $a_{\mu}$ [14]. However, for a determination of QCD condensates one does not need such a detailed, and generally model dependent analysis. In fact, isospin is almost exactly conserved in integrated cross 
sections or moments, provided the upper limit of the integration over squared energies energies, $s_{0}$, is taken large enough. This is because isospin-breaking terms are proportional to $m_{q}^{2} / s_{0}$ where $m_{q}$ refers to the mass of the light quarks. Therefore one can restrict the analysis of moments and condensates to the total $(I=1$ plus $I=0) e^{+} e^{-}$cross section.

A similar analysis based on the known $I=1$ spectral functions obtained from $\tau$-decay data has already been performed [5]-[8]. However, there the upper limit of integration $s_{0}$ is restricted by the $\tau$ mass to values of about $s_{0} \simeq 3 \mathrm{GeV}^{2}$ and it is not clear whether this value is large enough for PQCD to be applicable. In spite of this, the condensates derived from $\tau$-decay have been shown previously to be consistent with QCD expectations and we will therefore focus in the following on the case of $e^{+} e^{-}$annihilation.

The paper is organized as follows. In Section 2 we present a short summary of the theoretical background and in Section 3 we describe shortly the data used in our analysis, with a more detailed discussion deferred to the Appendix. Section 4 contains our results, their interpretation, and our conclusions.

\section{QCD Sum Rules}

We begin by considering the electromagnetic current correlator

$$
\begin{aligned}
\Pi_{\mu \nu}^{\mathrm{EM}}\left(q^{2}\right) & =i \int d^{4} x e^{i q x}\left\langle 0\left|T\left(J_{\mu}^{\mathrm{EM}}(x) J_{\nu}^{\mathrm{EM}}(0)\right)\right| 0\right\rangle \\
& =\left(-q^{2} g_{\mu \nu}+q_{\mu} q_{\nu}\right) \Pi^{\mathrm{EM}}\left(q^{2}\right),
\end{aligned}
$$

where for three flavours

$$
J_{\mu}^{\mathrm{EM}}(x)=\frac{2}{3} \bar{u}(x) \gamma^{\mu} u(x)-\frac{1}{3} \bar{d}(x) \gamma^{\mu} d(x)-\frac{1}{3} \bar{s}(x) \gamma^{\mu} s(x) .
$$

QCD is flavour blind, and if isospin invariance is exact, it is convenient to define a QCD current correlator using any of the quark currents $\bar{q}_{i} \gamma_{\mu} q_{i}$ with flavour $i$. This leads to

$$
\operatorname{Im} \Pi^{\mathrm{EM}}\left(q^{2}\right)=\sum_{i=1}^{n_{f}} Q_{i}^{2} \operatorname{Im} \Pi_{\mathrm{VV}}\left(q^{2}\right),
$$

where $Q_{i}$ is the charge of the quark $i=u, d, s, \ldots, \sum_{i} Q_{i}^{2}=2 / 3$ for $n_{f}=3$ flavours, and $\operatorname{Im} \Pi_{\mathrm{VV}}\left(q^{2}\right)$ is the QCD correlator of vector currents of flavour $i$. The electromagnetic spectral function, $\operatorname{Im} \Pi^{\mathrm{EM}}(s)$, with $s$ the square energy, is accessible experimentally from data on $e^{+} e^{-}$annihilation to hadrons as follows. The ratio $R(s)$ is defined as

$$
R(s)=\frac{\sigma_{\mathrm{TOT}}\left(e^{+} e^{-} \rightarrow \text { hadrons }\right)}{\sigma\left(e^{+} e^{-} \rightarrow \mu^{+} \mu^{-}\right)},
$$

with

$$
\sigma\left(e^{+} e^{-} \rightarrow \mu^{+} \mu^{-}\right)=\frac{4 \pi \alpha_{\mathrm{EM}}^{2}}{3 s}
$$


and $\alpha_{\mathrm{EM}}=e^{2} / 4 \pi$. In QCD the ratio $R$ and the electromagnetic spectral function are related through

$$
R(s)=12 \pi \operatorname{Im} \Pi^{\mathrm{EM}}(s)=3 \sum_{i=1}^{n_{f}} Q_{i}^{2}\left(1+\frac{\alpha_{s}}{\pi}+\ldots\right)
$$

A singlet contribution proportional to $\left(\sum_{i} Q_{i}\right)^{2}$ arises at order $\mathcal{O}\left(\alpha_{s}^{3}\right)$ and vanishes if one sums over three flavours. In particular, for the two-pion final state, dominated by the $\rho$-resonance, the relation between $R$ and the pion form factor, $F_{\pi}^{(0)}(s)$, is

$$
R_{e^{+} e^{-} \rightarrow \pi^{+} \pi^{-}}(s)=\frac{1}{4}\left(1-\frac{4 m_{\pi}^{2}}{s}\right)^{\frac{3}{2}}\left|F_{\pi}^{(0)}(s)\right|^{2} .
$$

On the QCD side, the vector correlator is assumed to satisfy the OPE extended beyond perturbation theory. Non-perturbative modifications due to confinement are parametrized in terms of vacuum condensates, i.e.,

$$
8 \pi \Pi_{\mathrm{VV}}\left(Q^{2}\right)=\sum_{N=0}^{\infty} \frac{1}{\left(Q^{2}\right)^{N}} C_{2 N}\left(Q^{2}, \mu^{2}\right)\left\langle 0\left|\mathcal{O}_{2 N}\left(\mu^{2}\right)\right| 0\right\rangle,
$$

where $Q^{2} \equiv-q^{2}>0$ is large, $\mu$ is a renormalization scale, and the first term with $N=0$ stands for the PQCD contribution. The Wilson coefficients $C_{2 N}$, calculable in perturbation theory, contain the short distance information while the vacuum condensates effectively parametrize the long distance dynamics. These condensates are organized according to their dimension, with the leading ones being the product of the quark mass and condensate of dimension $d=4, m_{q}\langle 0|\bar{q} q| 0\rangle$, and the gluon condensate also of $d=4$, $\left\langle 0\left|\frac{\alpha_{s}}{\pi} G_{\mu \nu} G^{\mu \nu}\right| 0\right\rangle$. This gives the result

$$
C_{4}\left\langle\mathcal{O}_{4}\right\rangle=\frac{\pi^{2}}{3}\left\langle\frac{\alpha_{s}}{\pi} G_{\mu \nu} G^{\mu \nu}\right\rangle+4 \pi^{2}\left(m_{u}\langle\bar{u} u\rangle+m_{d}\langle\bar{d} d\rangle+m_{s}\langle\bar{s} s\rangle\right)
$$

where $\alpha_{s}$ is the running strong coupling, and in the sequel $\left\langle 0\left|\mathcal{O}_{2 N}\right| 0\right\rangle \equiv\left\langle\mathcal{O}_{2 N}\right\rangle$ is to be understood. This condensate is renormalization group invariant to all orders in PQCD. Next, at dimension $d=6$ there enters the four-quark condensate

$$
\begin{aligned}
C_{6}\left\langle\mathcal{O}_{6}\right\rangle= & -4 \pi^{3}\left\langle\alpha_{s}\left(\bar{u} \gamma_{\mu} \gamma_{5} t^{a} u-\bar{d} \gamma_{\mu} \gamma_{5} t^{a} d\right)^{2}\right\rangle \\
& -\frac{8}{9} \pi^{3}\left\langle\alpha_{s}\left(\bar{u} \gamma_{\mu} t^{a} u+\bar{d} \gamma_{\mu} t^{a} d\right) \sum_{q=u, d, s} \bar{q} \gamma_{\mu} t^{a} q\right\rangle
\end{aligned}
$$

which has a mild dependence on the renormalization scale. A once popular approximation is that of vacuum saturation [2] which gives

$$
\left.C_{6}\left\langle\mathcal{O}_{6}\right\rangle\right|_{V S}=-\frac{896}{81} \pi^{3} \alpha_{s}|\langle\bar{q} q\rangle|^{2} \simeq-0.025 \mathrm{GeV}^{6}
$$

This value serves only as an order of magnitude reference, as there is no reliable way of estimating corrections to this approximation. In our analysis we do not need to invoke 
vacuum saturation as we will be determining the complete $C_{6}\left\langle\mathcal{O}_{6}\right\rangle$, independently of any theoretical assumption. While a dimension $d=2$ contribution is present in the PQCD term of the OPE for the vector correlator, it is numerically negligible as it is proportional to $m_{u}^{2}+m_{d}^{2}$.

The PQCD vector spectral function is well-known to five-loop order [15]-[19], i.e.

$$
\begin{aligned}
8 \pi \operatorname{Im} \Pi_{\mathrm{VV}}(s)= & 1+a_{s}+a_{s}^{2}\left(F_{3}+\frac{\beta_{1}}{2} L_{\mu}\right)+a_{s}^{3}\left[F_{4}+\left(\beta_{1} F_{3}+\frac{\beta_{2}}{2}\right) L_{\mu}+\frac{\beta_{1}^{2}}{4} L_{\mu}^{2}\right] \\
& +a_{s}^{4}\left[k_{3}-\frac{\pi^{2}}{4} \beta_{1}^{2} F_{3}-\frac{5}{24} \pi^{2} \beta_{1} \beta_{2}+\left(\frac{3}{2} \beta_{1} F_{4}+\beta_{2} F_{3}+\frac{\beta_{3}}{2}\right) L_{\mu}\right. \\
& \left.+\frac{\beta_{1}}{2}\left(\frac{3}{2} \beta_{1} F_{3}+\frac{5}{4} \beta_{2}\right) L_{\mu}^{2}+\frac{\beta_{1}^{3}}{8} L_{\mu}^{3}\right],
\end{aligned}
$$

where $a_{s} \equiv \alpha_{s}\left(\mu^{2}\right) / \pi, L_{\mu} \equiv \ln \left(Q^{2} / \mu^{2}\right), k_{3}=49.076$ [20], $F_{3}=1.9857-0.1153 n_{f}, F_{4}=$ $18.2427-\frac{\pi^{2}}{3}\left(\frac{\beta_{1}}{2}\right)^{2}-4.2158 n_{f}+0.0862 n_{f}^{2}, \beta_{1}=-\frac{1}{2}\left(11-\frac{2}{3} n_{f}\right), \beta_{2}=-\frac{1}{8}\left(102-\frac{38}{3} n_{f}\right)$, $\beta_{3}=-\frac{1}{32}\left(\frac{2857}{2}-\frac{5033}{18} n_{f}+\frac{325}{54} n_{f}^{2}\right)$, and the running coupling to four-loop order is [21]

$$
\begin{aligned}
\frac{\alpha_{s}^{(4)}\left(s_{0}\right)}{\pi}= & \frac{\alpha_{s}^{(1)}\left(s_{0}\right)}{\pi}+\left(\frac{\alpha_{s}^{(1)}\left(s_{0}\right)}{\pi}\right)^{2}\left(\frac{-\beta_{2}}{\beta_{1}} \ln L\right) \\
& +\left(\frac{\alpha_{s}^{(1)}\left(s_{0}\right)}{\pi}\right)^{3}\left(\frac{\beta_{2}^{2}}{\beta_{1}^{2}}\left(\ln ^{2} L-\ln L-1\right)+\frac{\beta_{3}}{\beta_{1}}\right) \\
& -\left(\frac{\alpha_{s}^{(1)}\left(s_{0}\right)}{\pi}\right)^{4}\left[\frac{\beta_{2}^{3}}{\beta_{1}^{3}}\left(\ln ^{3} L-\frac{5}{2} \ln ^{2} L-2 \ln L+\frac{1}{2}\right)\right. \\
& \left.+3 \frac{\beta_{2} \beta_{3}}{\beta_{1}^{2}} \ln L+\frac{b_{3}}{\beta_{1}}\right]
\end{aligned}
$$

with

$$
\frac{\alpha_{s}^{(1)}\left(s_{0}\right)}{\pi} \equiv \frac{-2}{\beta_{1} L},
$$

where $L \equiv \ln \left(s_{0} / \Lambda_{\overline{\mathrm{MS}}}^{2}\right)$ defines the standard $\overline{\mathrm{MS}}$ scale $\Lambda_{\overline{\mathrm{MS}}}$, and the constant $b_{3}$ is

$$
\begin{aligned}
b_{3}= & \frac{1}{4^{4}}\left[\frac{149753}{6}+3564 \zeta_{3}-\left(\frac{1078361}{162}+\frac{6508}{27} \zeta_{3}\right) n_{F}\right. \\
& \left.+\left(\frac{50065}{162}+\frac{6472}{81} \zeta_{3}\right) n_{F}^{2}+\frac{1093}{729} n_{F}^{3}\right]
\end{aligned}
$$

with $\zeta_{3}=1.202$. 
Invoking Cauchy's theorem in the complex squared energy s-plane allows to relate the experimentally measured hadronic spectral function with that from QCD (quark-hadron duality), leading to the finite energy sum rules (FESR)

$$
(-)^{N} C_{2 N+2}\left\langle\mathcal{O}_{2 N+2}\right\rangle=8 \pi^{2} \int_{0}^{s_{0}} d s s^{N} \frac{1}{\pi} \operatorname{Im} \Pi^{\mathrm{DATA}}(s)-s_{0}^{N+1} M_{2 N+2}\left(s_{0}\right),
$$

where the dimensionless PQCD moments $M_{2 N+2}\left(s_{0}\right)$ are given by

$$
\begin{aligned}
M_{2 N+2}\left(s_{0}\right) & \equiv-8 \pi^{2} \frac{1}{2 \pi i} \oint_{C\left(\left|s_{0}\right|\right)} \frac{d s}{s_{0}}\left[\frac{s}{s_{0}}\right]^{N} \Pi^{\mathrm{PQCD}}(s) \\
& =8 \pi^{2} \int_{0}^{s_{0}} \frac{d s}{s_{0}}\left[\frac{s}{s_{0}}\right]^{N} \frac{1}{\pi} \operatorname{Im} \Pi(s)^{\mathrm{PQCD}} .
\end{aligned}
$$

It has been assumed that $s_{0}$ is large enough so that the replacement $\Pi(s) \rightarrow \Pi^{\mathrm{PQCD}}(s)$ is justified on the circle of radius $\left|s_{0}\right|$ in the complex $s$-plane. It must be mentioned that there is no mixing of operators of different dimension up to second-loop order in the coupling [22], hence each FESR involves only one condensate. We shall neglect radiative corrections to the condensates and proceed to determine the condensates of dimension $d=2$ to $d=6$ using the FESR, Eq. (16). This approximation is justified a-posteriori, given the resulting large errors in the condensates.

The contour integral in the complex s-plane will be evaluated in the framework of Fixed Order Perturbation Theory (FOPT), as well as in Contour Improved Perturbation Theory (CIPT). In FOPT the coupling and the quark masses at a scale $s_{0}$ are considered fixed (constant) so that only logarithmic terms contribute to the integral. The renormalization group ( $\mathrm{RG}$ ) summation of leading logs is only carried out after the contour integration by setting $\mu^{2}=-s_{0}$. In this case the integration can be done analytically. In the case of CIPT the strong coupling and the quark masses are running and the RG is implemented before integrating. The RG equation for the running coupling and quark masses is solved numerically at each point on the circle of radius $s_{0}$. The value of the strong coupling to be used here is $\alpha_{s}\left(M_{\tau}^{2}\right)=0.321 \pm 0.015$, corresponding to $\Lambda_{\overline{\mathrm{MS}}}=341 \pm 24 \mathrm{MeV}$ in FOPT, or $\alpha_{s}\left(M_{\tau}^{2}\right)=0.344 \pm 0.014$ corresponding to $\Lambda_{\overline{\mathrm{MS}}}=382 \pm 24 \mathrm{MeV}$ in CIPT [23]. If one were to compare results for the condensates obtained from $e^{+} e^{-}$data as described above with corresponding results from $\tau$-decay, one would need to change the overall normalization of the QCD correlator.

\section{Hadronic Data}

The most rigorous approach to making a collection of exclusive data is to combine all available data for a given final state [24]-[51]. Their respective statistical and systematic uncertainties should be used to define the weight in the averaging procedure. In fact, this is important for precision determinations, such as, e.g., the $g-2$ factor of the muon. However, one can be less rigorous for the purpose of the present analysis. The philosophy 


\begin{tabular}{|c|c|c|c|}
\hline Number & $e^{+} e^{-} \rightarrow$ & $\mathcal{I}_{0} \times 10^{2}\left(\mathrm{GeV}^{2}\right)$ & $\mathcal{I}_{1} \times 10^{2}\left(\mathrm{GeV}^{4}\right)$ \\
\hline 1 & $\pi^{0} \gamma$ & $1.51(4)(9)$ & $0.99(3)(6)$ \\
\hline 2 & $\eta \gamma$ & $0.30(4)(2)$ & $0.29(5)(2)$ \\
\hline 3 & $\pi^{+} \pi^{-}$ & $133.1(25)(8)$ & $95.6(27)(7)$ \\
\hline 4 & $\pi^{+} \pi^{-} \pi^{0}$ & $30.1(3)(14)$ & $41.6(8)(16)$ \\
\hline 5 & $2\left(\pi^{+} \pi^{-}\right)$ & $60.5(3)(30)$ & $156.6(10)(78)$ \\
\hline 6 & $\pi^{+} \pi^{-} 2\left(\pi^{0}\right)$ & $79.6(4)(69)$ & $206.6(13)(181)$ \\
\hline 7 & $2 \pi^{+} 2 \pi^{-} \pi^{0}$ & $7.1(2)(7)$ & $20.8(6)(20)$ \\
\hline 8 & $3\left(\pi^{+} \pi^{-}\right)$ & $2.59(7)(2)$ & $8.83(30)(60)$ \\
\hline 9 & $2\left(\pi^{+} \pi^{-} \pi^{0}\right)$ & $11.6(3)(12)$ & $39.2(10)(41)$ \\
\hline 10 & $\eta\left(\pi^{+} \pi^{-}\right)$ & $6.9(3)(6)$ & $18.9(8)(16)$ \\
\hline 11 & $\eta \omega$ & $3.1(1)(3)$ & $9.0(3)(9)$ \\
\hline 12 & $\eta\left(2 \pi^{+} 2 \pi^{-}\right)$ & $1.0(1)(1)$ & $3.6(4)(3)$ \\
\hline 13 & $\omega \pi^{0}\left(\omega \rightarrow \pi^{0} \gamma\right)$ & $2.22(4)(6)$ & $4.5(1)(1)$ \\
\hline 14 & $K^{+} K^{-}$ & $25.2(3)(6)$ & $34.0(7)(9)$ \\
\hline 15 & $K_{s}^{0} K_{L}^{0}$ & $12.2(2)(5)$ & $14.2(5)(7)$ \\
\hline 16 & $\eta \dot{\phi}$ & $3.6(2)(3)$ & $11.5(7)(8)$ \\
\hline 17 & $p \bar{p}$ & $0.82(3)(4)$ & $3.0(1)(2)$ \\
\hline 18 & $n \bar{n}$ & $0.9(3)(1)$ & $3.5(13)(5)$ \\
\hline 19 & Inclusive & $56.0(4)(2)$ & $231.6(30)(9)$ \\
\hline $20^{*}$ & $\pi^{+} \pi^{-}(\mathrm{CHPT})$ & $0.01(0)(0)$ & $0.001(0)(0)$ \\
\hline $21^{*}$ & $\pi^{+} \pi^{-} 3 \pi^{0}$ & $3.5(1)(12)$ & $10.4(3)(34)$ \\
\hline $22^{*}$ & $\pi^{+} \pi^{-}\left(4 \pi^{0}\right)$ & $1.9(1)(6)$ & $6.2(2)(21)$ \\
\hline $23^{*}$ & $\omega \pi^{+} \pi^{-}, \omega 2 \pi^{0}\left(\omega \rightarrow \pi^{0} \gamma\right)$ & $0.56(2)(18)$ & $1.6(1)(5)$ \\
\hline $24^{*}$ & $\omega($ non- $3 \pi, \pi \gamma, \eta \gamma)$ & $0.11(1)(4)$ & $0.07(1)(2)$ \\
\hline $25^{*}$ & $\phi($ non- $K \bar{K}, 3 \pi, \pi \gamma, \eta \gamma)$ & $0.04(0)(1)$ & $0.04(0)(1)$ \\
\hline $26^{*}$ & $\eta \pi^{+} \pi^{-}\left(2 \pi^{0}\right)$ & $1.0(1)(3)$ & $3.6(4)(12)$ \\
\hline $27^{*}$ & $K \bar{K} \pi$ & $16.6(4)(57)$ & $47(12)(16)$ \\
\hline $28^{*}$ & $K \bar{K} 2 \pi$ & $36.1(9)(64)$ & $124(3)(22)$ \\
\hline \multirow[t]{2}{*}{$29^{*}$} & $K \bar{K} 3 \pi$ & $1.7(1)(3)$ & $6.1(5)(10)$ \\
\hline & Total & $499(3)(13)$ & $1105(5)(38)$ \\
\hline
\end{tabular}

Table 1: The contributions to $\mathcal{I}_{n}=\frac{1}{2} \int_{0}^{s_{0}} s^{n} R(s) d s$, using $s_{0}=4.5 \mathrm{GeV}^{2}$. The first error is statistical, while the second is systematic. For the total uncertainty, we added the uncertainties of all the final states in quadrature. Stars $(*)$ indicate that the cross section had to be estimated, following the prescriptions explained in Appendix A. 


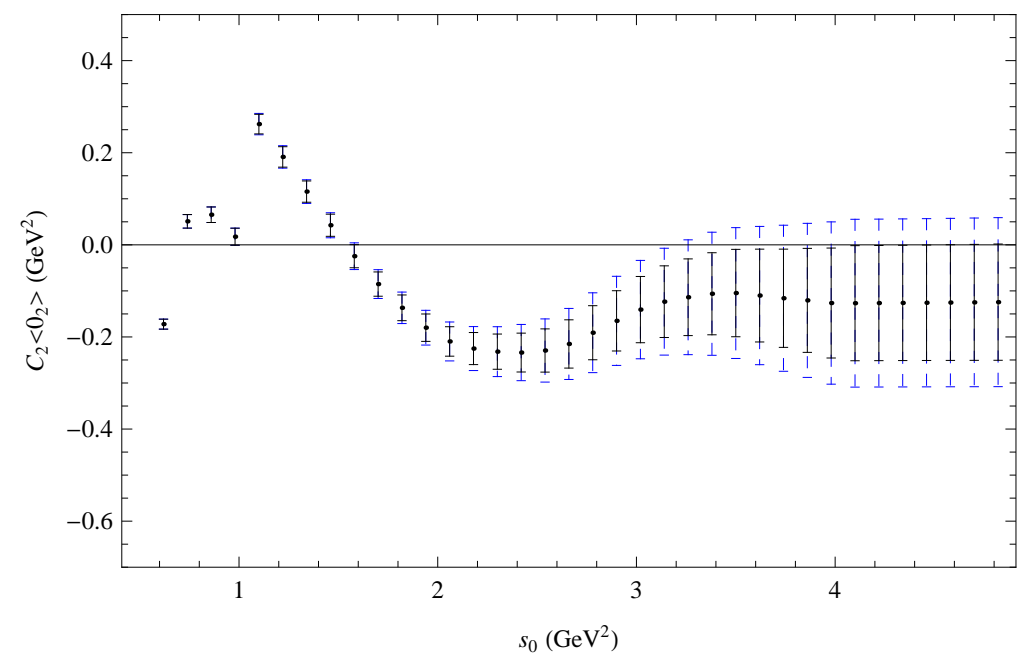

Figure 1: $\quad C_{2}\left\langle\mathcal{O}_{2}\right\rangle$ calculated in FOPT using $\alpha_{s}\left(M_{\tau}^{2}\right)=0.321 \pm 0.015$, corresponding to $\Lambda_{\frac{\left(n_{f}=3\right)}{\mathrm{MS}}}=341$ $\mathrm{MeV}$. The smaller uncertainties are obtained assuming no correlations between experiments, while the larger ones assume $100 \%$ correlations for data obtained using the same experimental facility.

is to make use of only the most recent data for a given exclusive hadronic final state. If the most recent data do not cover the entire energy range for that final state, then older data are used to cover this region. In this way there are no overlapping data sets, and then one does not need to be concerned about how precisely different, often inconsistent, data are combined. We will make use of exclusive data up to $\sqrt{s}=2 \mathrm{GeV}$, and above this energy we use the BES inclusive data [45]-[46]. These data are consistent with PQCD, hence one observes a plateau for the condensates above $\sqrt{s_{0}}=2 \mathrm{GeV}$. Since, in addition, their systematic errors are small $(3 \%)$ we observe that the resulting errors for $C_{N}\left\langle\mathcal{O}_{N}\right\rangle$ do not change any more above this energy. The treatment of the statistical uncertainties is standard. We assume no correlations either within individual experiments or between different experiments. The exception to this is the treatment of the $\pi^{+} \pi^{-}$ data from BaBar [25]. In this case we are given a full correlation matrix. To simplify the calculation, we simply assumed $100 \%$ correlation for the statistical uncertainty, which gives an upper bound on the uncertainty 2 For the systematic errors, we assume that they are completely correlated within a data set. It is reasonable to assume that there will also be correlations between different final-state data sets obtained by the same experimental facility, e.g. BaBar, CMD2 or SND, since each experiment would use the same procedure when applying radiative corrections or for the determination of the luminosity. As we do not have any information about these correlations, we will quote our final uncertainty both for the worst-case scenario assuming a $100 \%$ correlation of data determined at the same facility, as well as for the best-case scenario of no correlations. The true uncertainty can be taken to lie between these two cases. For more details see the Appendix where we also present the list of all final states used in our analysis in Table 2. We have verified our

\footnotetext{
${ }^{2}$ We also checked that this assumption does not have a significant impact on the uncertainty in the final results for the condensates.
} 


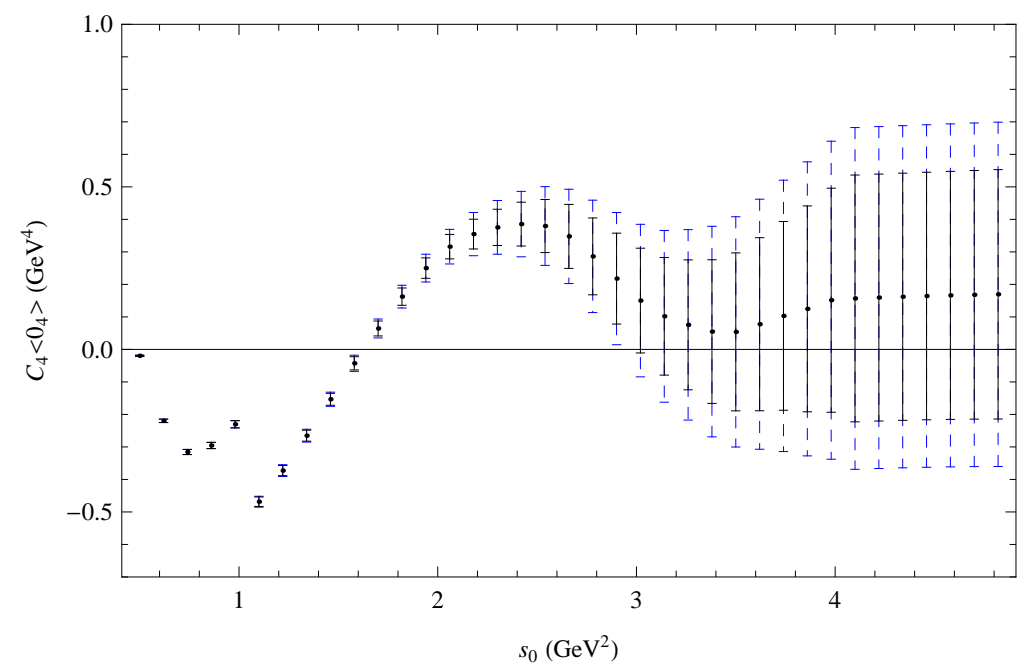

Figure 2: $\quad C_{4}\left\langle\mathcal{O}_{4}\right\rangle$ calculated in FOPT using $\alpha_{s}\left(M_{\tau}^{2}\right)=0.321 \pm 0.015$, corresponding to $\Lambda_{\overline{\mathrm{MS}}}^{\left(n_{f}=3\right)}=341$ $\mathrm{MeV}$. The smaller uncertainties are obtained assuming no correlations between experiments, while the larger ones assume $100 \%$ correlations for data obtained using the same experimental facility.

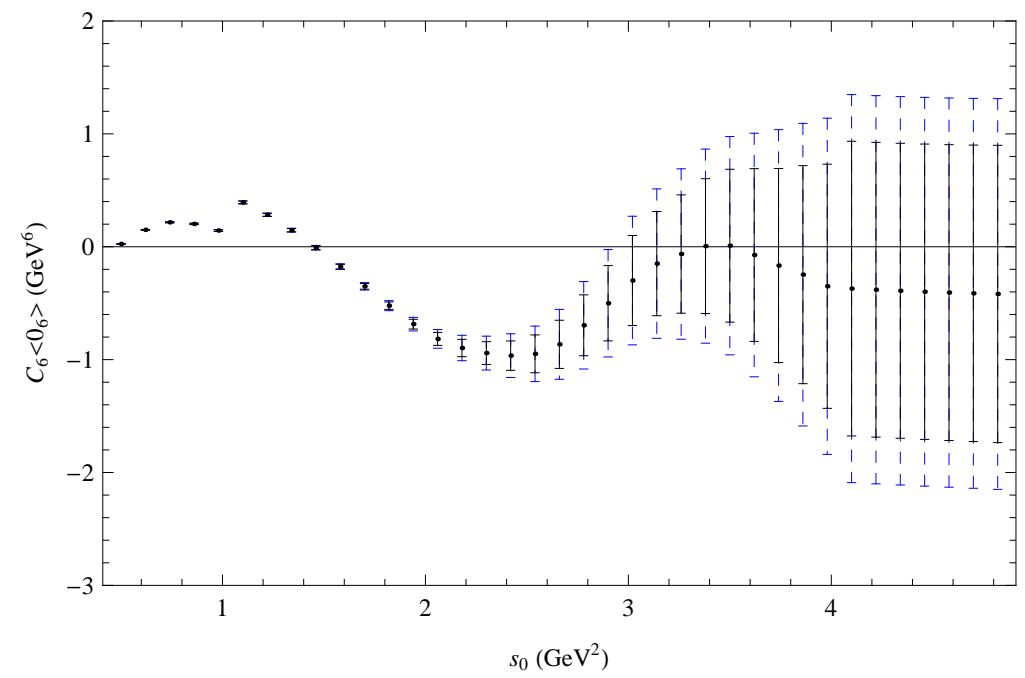

Figure 3: $\quad C_{6}\left\langle\mathcal{O}_{6}\right\rangle$ calculated in FOPT using $\alpha_{s}\left(M_{\tau}^{2}\right)=0.321 \pm 0.015$, corresponding to $\Lambda_{\mathrm{MS}}^{\left(n_{f}=3\right)}=341$ $\mathrm{MeV}$. The smaller uncertainties are obtained assuming no correlations between experiments, while the larger ones assume $100 \%$ correlations for data obtained using the same experimental facility.

data base against the one used in [11] for the determination of the muon anomaly, $a_{\mu}$. 


\section{Results and Conclusions}

Results for the condensates of the total $I=0$ plus $I=1$ current correlator determined from $e^{+} e^{-}$data are presented in Figs. 1,3. From standard QCD and the OPE one would expect the dimension $d=2$ condensate to be zero. Fig. 1 shows that this is the case within (large) errors, although the central value is negative. It is also seen from this figure that the sum rule starts to be saturated only at about $s_{0} \approx 3.4 \mathrm{GeV}^{2}$. For the condensate of dimension $d=4$ shown in Fig. 2 we observe saturation of the sum rule at a slightly higher $s_{0}$ than in the case of dimension $d=2$. The central value of the dimension $d=4$ condensate is seen to be mostly positive, as it should be since it is related to the vacuum energy. Figure 3 shows the result for the dimension $d=6$ condensate. Since the errors are so large, no meaningful conclusion can be drawn in this case, other than that the central value is negative, in agreement with the vacuum saturation approximation, Eq. (11). The results for the condensates are not very sensitive to the value of $\alpha_{s}$ used in the sum rules. Their values at $s_{0}=4.5 \mathrm{GeV}^{2}$, assuming uncorrelated data errors are

$$
\begin{aligned}
& C_{2}\left\langle\mathcal{O}_{2}\right\rangle=\left\{\begin{array}{l}
\left.\left(-0.13 \pm\left. 0.13\right|_{\mathrm{EXP}} \pm\left. 0.03\right|_{\alpha_{s}}\right) \mathrm{GeV}^{2}\right|_{\mathrm{FOPT}} \\
\left.\left(-0.14 \pm\left. 0.13\right|_{\mathrm{EXP}} \pm\left. 0.03\right|_{\alpha_{s}}\right) \mathrm{GeV}^{2}\right|_{\mathrm{CIPT}}
\end{array}\right. \\
& C_{4}\left\langle\mathcal{O}_{4}\right\rangle=\left\{\begin{array}{l}
\left.\left(+0.16 \pm\left. 0.38\right|_{\mathrm{EXP}} \pm\left. 0.04\right|_{\alpha_{s}}\right) \mathrm{GeV}^{4}\right|_{\mathrm{FOPT}} \\
\left.\left(+0.10 \pm\left. 0.38\right|_{\mathrm{EXP}} \pm\left. 0.04\right|_{\alpha_{s}}\right) \mathrm{GeV}^{4}\right|_{\mathrm{CIPT}}
\end{array}\right. \\
& C_{6}\left\langle\mathcal{O}_{6}\right\rangle=\left\{\begin{array}{l}
\left.\left(-0.40 \pm\left. 1.30\right|_{\mathrm{EXP}} \pm\left. 0.10\right|_{\alpha_{s}}\right) \mathrm{GeV}^{6}\right|_{\mathrm{FOPT}} \\
\left.\left(-0.40 \pm\left. 1.30\right|_{\mathrm{EXP}} \pm\left. 0.10\right|_{\alpha_{s}}\right) \mathrm{GeV}^{6}\right|_{\mathrm{CIPT}}
\end{array}\right.
\end{aligned}
$$

Assuming instead maximally correlated data errors the experimental uncertainties above increase to $\pm\left. 0.18\right|_{\mathrm{EXP}}, \pm\left. 0.52\right|_{\mathrm{EXP}}$, and $\pm\left. 1.70\right|_{\mathrm{EXP}}$, for $C_{2}\left\langle\mathcal{O}_{2}\right\rangle, C_{4}\left\langle\mathcal{O}_{4}\right\rangle$, and $C_{6}\left\langle\mathcal{O}_{6}\right\rangle$, respectively. These results are consistent with expectations and with the values extracted from $\tau$-decay [5]-[8], albeit within very large errors. The uncertainties in all the results are clearly dominated by the experimental errors. One consequence of this is that e.g. in the $d=2$ case it is not possible to distinguish among various theoretical sources of such a term in the OPE. This is also the case with the result from the $\tau$ decay data [5]-[6], although it has a smaller uncertainty. In any case, the impact of the PQCD input for a given integration method (FOPT or CIPT) is such that the higher the value of $\alpha_{s}$ the lower the curve in Fig.1. A similar trend is found from additional higher order PQCD terms, and this behaviour is common to all condensates. For the gluon condensate the value determined from charmonium data is $C_{4}\left\langle\mathcal{O}_{4}\right\rangle=0.06 \pm 0.04 \mathrm{GeV}^{4}$ [2], [52]-55], where the error is our conservative estimate. The result from $\tau$-decay is [6] $C_{4}\left\langle\mathcal{O}_{4}\right\rangle=0.07 \pm 0.02 \mathrm{GeV}^{4}$ for a similar input value of $\alpha_{s}$. Concerning the $d=6$ result, given the large uncertainty in its value no meaningful comparison can be made with e.g. the vacuum saturation prediction, Eq.(11). It should be emphasized that the consistency between data and QCD is 


\begin{tabular}{|c|c|c|c|c|}
\hline Number & $e^{+} e^{-} \rightarrow$ & Reference & $\begin{array}{c}\text { Data Range } \\
\sqrt{s}(\mathrm{GeV})\end{array}$ & Vacuum Pol. \\
\hline 1 & $\pi^{0} \gamma$ & CMD-2 (2005) 24 & $0.6-1.31$ & Dressed \\
\hline 2 & $\eta \gamma$ & CMD-2 (2005) 24 & $0.6-1.38$ & Dressed \\
\hline 3 & $\pi^{+} \pi^{-}$ & BaBar (2009) 25] & $0.31-2.95$ & Bare \\
\hline \multirow[t]{4}{*}{4} & $\pi^{+} \pi^{-} \pi^{0}$ & BaBar (2004) 26] & $1.06-2.99$ & Dressed \\
\hline & & CMD-2 (2006) 27. & $1.01-1.06$ & Dressed \\
\hline & & SND (2002) 28] & $0.98-1.01$ & Dressed \\
\hline & & SND (2003) 29] & $0.66-0.98$ & Dressed \\
\hline 5 & $2\left(\pi^{+} \pi^{-}\right)$ & BaBar (2005) 30 & $0.62-4.45$ & Bare \\
\hline \multirow[t]{2}{*}{6} & $\pi^{+} \pi^{-} 2\left(\pi^{0}\right)$ & BaBar (2010) 31 & $0.76-3.31$ & Dressed \\
\hline & & SND (2009) 32 & $0.66-0.94$ & Dressed \\
\hline 7 & $2 \pi^{+} 2 \pi^{-} \pi^{0}$ & BaBar (2007) 33 & $1.03-2.98$ & Dressed \\
\hline 8 & $3\left(\pi^{+} \pi^{-}\right)$ & BaBar (2006) 34 & $1.3-4.5$ & Dressed \\
\hline 9 & $2\left(\pi^{+} \pi^{-} \pi^{0}\right)$ & $\operatorname{BaBar}(2006) 34$ & $1.3-4.5$ & Dressed \\
\hline 10 & $\eta\left(\pi^{+} \pi^{-}\right)$ & BaBar (2007) 33 & $1.03-2.98$ & Dressed \\
\hline 11 & $\eta \omega$ & $\operatorname{BaBar}(2006) 34$ & $1.25-3.25$ & Dressed \\
\hline 12 & $\eta\left(2 \pi^{+} 2 \pi^{-}\right)$ & BaBar (2007) 33 & $1.31-2.89$ & Dressed \\
\hline 13 & $\omega \pi^{0}\left(\omega \rightarrow \pi^{0} \gamma\right)$ & CMD-2 (2003) 35 & $0.92-1.38$ & Bare \\
\hline \multirow[t]{3}{*}{14} & $K^{+} K^{-}$ & CMD-2 (2008) 36 & $1.011-1.034$ & Dressed \\
\hline & & SND (2007) 37 & $1.04-1.38$ & Dressed \\
\hline & & DM2 (1988) 38 & $1.38-2.40$ & $?$ \\
\hline \multirow[t]{3}{*}{15} & $K_{s}^{0} K_{L}^{0}$ & SND (2001) 39 & $1.01-1.06$ & Dressed \\
\hline & & SND (2006) 40 & $1.04-1.38$ & Dressed \\
\hline & & DM1 (1981) 41] & $1.4-2.1$ & $?$ \\
\hline 16 & $\eta \phi$ & BaBar (2008) 42 & $1.57-3.45$ & Dressed \\
\hline 17 & $p \bar{p}$ & BaBar (2006) 43 & $1.88-4.2$ & Dressed \\
\hline 18 & $n \bar{n}$ & Fenice (1998) 44] & $1.9-2.44$ & Dressed \\
\hline \multirow[t]{2}{*}{19} & Inclusive & BES (2002) 45] & $2-5$ & Bare \\
\hline & & BES (2009) 46 & $2.6-3.65$ & Bare \\
\hline 20 & $\pi^{+} \pi^{-}(\chi \mathrm{pT})$ & $*$ & $0.14-0.31$ & \\
\hline 21 & $\pi^{+} \pi^{-} 3 \pi^{0}$ & $*$ & $1.03-2.98$ & \\
\hline 22 & $\pi^{+} \pi^{-}\left(4 \pi^{0}\right)$ & $*$ & $1.3-4.5$ & \\
\hline 23 & $\omega \pi^{+} \pi^{-}, \omega 2 \pi^{0}\left(\omega \rightarrow \pi^{0} \gamma\right)$ & $*$ & $1.15-2.53$ & \\
\hline 24 & $\omega($ non- $3 \pi, \pi \gamma, \eta \gamma)$ & $*$ & $0.7-0.8$ & \\
\hline 25 & $\phi($ non- $K \bar{K}, 3 \pi, \pi \gamma, \eta \gamma)$ & $*$ & $1.01-1.03$ & \\
\hline 26 & $\eta \pi^{+} \pi^{-}\left(2 \pi^{0}\right)$ & $*$ & $1.3-2.9$ & \\
\hline 27 & $K \bar{K} \pi$ & $*$ & $1.3-4.7$ & \\
\hline 28 & $K \bar{K} 2 \pi$ & $*$ & $1.4-4.3$ & \\
\hline 29 & $K \bar{K} 3 \pi$ & $*$ & $1.6-4.5$ & \\
\hline
\end{tabular}

Table 2: Data used in evaluating the condensates. Stars $(*)$ indicate that the final state has not been measured and was estimated as explained in the text. The treatment of the vacuum polarization correction is described in the text. Question marks indicate that it is not known which corrections have been applied by the experimental collaboration, and no corrections were applied by us in these cases. 
in no way obvious. The high sensitivity of the FESR can be demonstrated if one were to determine condensates from the individual $I=0$ and $I=1$ channels separately. For example, for the non-strange $I=1$ final states listed in lines $2,3,5,6,8-10,12$, 22, and 26 of Tables 1 and 2 , the dimension $d=2$ and $d=4$ condensates would turn out to be unacceptably large, i.e. more than $3 \sigma$ away from theoretical expectations. Moreover, with this subset of data there would no longer be a clear plateau in the $s_{0}$-dependence , as the one in Figs. 1, 2 and 3. We believe that these unacceptable results indicate that the separation of the data into $I=1$ and $I=0$ components requires a fairly detailed understanding of isospin symmetry breaking. If isospin symmetry is broken, states with different isospin quantum numbers can mix and unknown interference contributions can spoil a naive decomposition into a sum of isovector plus isoscalar parts.

To summarize, with the help of FESR we have confronted QCD and the OPE with experimental data for $R(s)$. In particular we have considered the zeroth, first and second moment of $R(s)$. This comparison of theory with experiment can simultaneously help to give support to the theoretical framework, i.e. the basic principles of QCD, the OPE and analyticity, and as a test of the quality and completeness of the data. We found that recent $e^{+} e^{-}$annihilation data are consistent with QCD and the OPE within very large errors, even though there exists some indication that not all data are yet correctly accounted for. Any future improvement on the accuracy of vacuum condensate determinations from $e^{+} e^{-}$annihilation will require a considerable reduction in the experimental uncertainties.

\section{A Hadronic Data}

In this Appendix we give details of the data sets used in our analysis. Table 2 lists all relevant final states, their corresponding energy ranges and the corresponding references. Some of the exclusive channels are as yet unmeasured. We give here the estimated values of these missing channels. These estimates are mostly based on isospin arguments, primarily as described in Refs. [11] and [47, where further details can be found. It should be mentioned that the Pais isospin-class analysis [11] can be employed only after removing the $\eta$-contribution from some of the final states. This also avoids double counting in the evaluation of the FESR integrals. We have only quoted the results. A $33 \%$ model error has been assigned to all of these data, unless stated otherwise. The numbers in the following list refer to the final states listed in Table 2.

20. There are no data for the $\pi^{+} \pi^{-}$final state at low energies, so the prediction of chiral perturbation theory (CHPT) has been used. The cross section is expressed in terms of the $\pi$ form factor, $\sigma\left(\pi^{+} \pi^{-}\right)=\pi \alpha^{2} \beta_{0}^{3}\left|F_{\pi}^{0}\right|^{2} /(3 s)$, where $\beta_{0}=\left(1-4 m_{\pi}^{2} / s\right)^{1 / 2}$. The form factor is taken of the form $F_{\pi}^{0}=1+\frac{1}{6}\left\langle r^{2}\right\rangle_{\pi} s+c_{1} s^{2}+c_{2} s^{3}+\mathcal{O}\left(s^{4}\right)$. The constants are found by fitting the form factor to space-like data, with the result $\left\langle r^{2}\right\rangle_{\pi}=(0.439 \pm 0.008) \mathrm{fm}^{2}, c_{1}=(6.8 \pm 1.9) \mathrm{GeV}^{-4}$ and $c_{2}=(-0.7 \pm 6.8) \mathrm{GeV}^{-8}$ 47. 
21. A prediction for the missing channel $\sigma\left(\pi^{+} \pi^{-} 3 \pi^{0}\right)_{\eta \text {-excl. }}=\frac{1}{2} \sigma\left(2 \pi^{+} 2 \pi^{-} \pi^{0}\right)_{\eta \text {-excl. }}$ can be obtained after removing the $\eta$-contribution: $\sigma\left(2 \pi^{+} 2 \pi^{-} \pi^{0}\right)_{\eta \text {-excl. }}=\sigma\left(2 \pi^{+} 2 \pi^{-} \pi^{0}\right)-$ $\sigma\left(\eta \pi^{+} \pi^{-}\right) \times \mathcal{B}\left(\eta \rightarrow \pi^{+} \pi^{-} \pi^{0}\right)$, where $\mathcal{B}\left(\eta \rightarrow \pi^{+} \pi^{-} \pi^{0}\right)=0.2274 \pm 0.0028$ and where $\sigma\left(2 \pi^{+} 2 \pi^{-} \pi^{0}\right)$ is obtained from BaBar (2007) [33].

22. $\sigma\left(\pi^{+} \pi^{-} 4 \pi^{0}\right)=0.0625 \sigma\left(3 \pi^{+} 3 \pi^{-}\right)+0.145 \sigma\left(2 \pi^{+} 2 \pi^{-} 2 \pi^{0}\right)_{\eta-\text { excl }}$ from isospin symmetry, where the $\eta$-subtracted cross section $\sigma\left(2 \pi^{+} 2 \pi^{-} 2 \pi^{0}\right)_{\eta \text {-excl }}$ is determined using $\sigma\left(2 \pi^{+} 2 \pi^{-} 2 \pi^{0}\right)_{\eta \text {-excl. }}=\sigma\left(2 \pi^{+} 2 \pi^{-} 2 \pi^{0}\right)-\sigma(\eta \omega) \times \mathcal{B}\left(\eta \rightarrow \pi^{+} \pi^{-} \pi^{0}\right) \times \mathcal{B}\left(\omega \rightarrow \pi^{+} \pi^{-} \pi^{0}\right)$, where $\mathcal{B}\left(\omega \rightarrow \pi^{+} \pi^{-} \pi^{0}\right)=0.892 \pm 0.007, \sigma\left(2 \pi^{+} 2 \pi^{-} 2 \pi^{0}\right)$ was measured by BaBar [34], and $\sigma(\eta \omega)$ was taken from [34].

23. One has $\sigma\left(\omega \pi^{+} \pi^{-}\right)$from BaBar (2007) [33]. However, the dominant three-pion decay of the $\omega$ already appears in the five-pion final state. Thus we calculate the contribution for $\omega \rightarrow \pi^{0} \gamma$ by using $\sigma\left(\omega \pi^{+} \pi^{-} \rightarrow \pi^{+} \pi^{-} \pi^{0} \gamma\right)=\sigma\left(\omega \pi^{+} \pi^{-}\right) \times \mathcal{B}(\omega \rightarrow$ $\left.\pi^{0} \gamma\right)$ where $\mathcal{B}\left(\omega \rightarrow \pi^{0} \gamma\right)=0.0828 \pm 0.0028$. Then from isospin arguments it follows that $\sigma\left(\omega 2 \pi^{0} \rightarrow 3 \pi^{0} \gamma\right)=0.5 \sigma\left(\omega \pi^{+} \pi^{-} \rightarrow \pi^{+} \pi^{-} \pi^{0} \gamma\right)$.

24. There are cross section data for $\sigma\left(\omega \rightarrow \pi^{+} \pi^{-} \pi^{0}\right)$ from [48]. This is the major decay mode of the $\omega$, with $\mathcal{B}\left(\omega \rightarrow \pi^{+} \pi^{-} \pi^{0}\right)=0.892 \pm 0.007$. In addition to this final state, we have already accounted for the $\pi^{0} \gamma, \pi^{+} \pi^{-}$, and $\eta \gamma$ final states. We thus estimate the contribution from $\omega$ decays not yet accounted for as being $\sigma\left(\omega \rightarrow \pi^{+} \pi^{-} \pi^{0}\right) \times$ $\mathcal{B}($ non $-3 \pi, \pi \gamma, \eta \gamma) / \mathcal{B}\left(\omega \rightarrow \pi^{+} \pi^{-} \pi^{0}\right)$, where $\mathcal{B}($ non $-3 \pi, \pi \gamma, \eta \gamma)=0.0094$.

25. We have already accounted for $\phi$ to $K \bar{K}, 3 \pi, \pi \gamma$ and $\eta \gamma$. Hence there is a missing contribution found from $\mathcal{B}($ missing $)=1-\mathcal{B}(\phi \rightarrow K \bar{K})-\mathcal{B}(\phi \rightarrow 3 \pi)-\mathcal{B}(\phi \rightarrow$ $\pi \gamma)-\mathcal{B}(\phi \rightarrow \eta \gamma)=0.0014$. We then calculate $\sigma(\phi($ non- $K \bar{K}, 3 \pi, \pi \gamma, \eta \gamma))=\sigma(\phi \rightarrow$ $\left.K^{+} K^{-}\right) \mathcal{B}($ missing $) / \mathcal{B}\left(\phi \rightarrow K^{+} K^{-}\right)=0.003 \sigma\left(\phi \rightarrow K^{+} K^{-}\right)$.

26. We assume that $\sigma\left(\eta 2 \pi^{+} 2 \pi^{-}\right)=\sigma\left(\eta \pi^{+} \pi^{-} 2 \pi^{0}\right)$.

27. One has $\sigma(K \bar{K} \pi)=3 \sigma\left(K_{s}^{0} K^{ \pm} \pi^{\mp}\right)+\sigma\left(\phi \pi^{0}\right) \times \mathcal{B}(\phi \rightarrow K \bar{K})$. Here $\sigma\left(K_{s}^{0} K^{ \pm} \pi^{\mp}\right)$ and $\sigma\left(\phi \pi^{0}\right)$ are obtained from BaBar (2008) [42]. In addition, $\mathcal{B}(\phi \rightarrow K \bar{K})=$ $0.831 \pm 0.003$.

28. $\sigma(K \bar{K} 2 \pi)=9 \sigma\left(K^{+} K^{-} 2 \pi^{0}\right)+\frac{9}{4} \sigma\left(K^{+} K^{-} \pi^{+} \pi^{-}\right)$. The cross sections for the $K^{+} K^{-} 2 \pi$ final states are available from BaBar (2007) [49]. To estimate an uncertainty for this result we make use of a different procedure from [50] which uses the inclusive data $K_{S} X$ (DM1 Collaboration, [51]) as a starting point. The difference in cross sections between these methods is $17 \%$, which we take to be the systematic uncertainty.

29. We assume that $\sigma\left(K^{0} \bar{K}^{0} \pi^{+} \pi^{-} \pi^{0}\right)_{\eta \text {-excl }}=\sigma\left(K^{+} K^{-} \pi^{+} \pi^{-} \pi^{0}\right)_{\eta-\text { excl }}$. With these two processes as primary contributors we find $\sigma(K \bar{K} 3 \pi)=2 \sigma\left(K^{+} K^{-} \pi^{+} \pi^{-} \pi^{0}\right)_{\eta-\text { excl }}$. We calculate $\sigma\left(K^{+} K^{-} \pi^{+} \pi^{-} \pi^{0}\right)_{\eta-\text { excl }}=\sigma\left(K^{+} K^{-} \pi^{+} \pi^{-} \pi^{0}\right)-\sigma(\phi \eta) \times \mathcal{B}\left(\phi \rightarrow K^{+} K^{-}\right) \times$ $\mathcal{B}\left(\eta \rightarrow \pi^{+} \pi^{-} \pi^{0}\right)$, where $\mathcal{B}\left(\phi \rightarrow K^{+} K^{-}\right)=0.489 \pm 0.01$ and $\mathcal{B}\left(\eta \rightarrow \pi^{+} \pi^{-} \pi^{0}\right)=$ $0.2274 \pm 0.0028$. We obtain $\sigma\left(K^{+} K^{-} \pi^{+} \pi^{-} \pi^{0}\right)$ from BaBar (2007) [33], and $\sigma(\phi \eta)$ is taken from BaBar (2008) [42]. 
When using the cross sections in the sum rules one needs the so-called undressed (or bare) cross-sections, $\sigma_{0}$. The bare cross sections are obtained by removing initial-state radiation, but not final-state radiation, and the contribution due to vacuum polarization. The leptonic part of the vacuum polarization has been subtracted already from the BaBar, SND, and CMD2 data. However, most of the data have not been corrected for the hadronic vacuum polarization. We do this by using

$$
\sigma_{0}=\left(\frac{\alpha(0)}{\alpha(s)}\right)^{2} \sigma_{\mathrm{born}}=\left|1-\Pi^{\prime}(s)\right|^{2} \sigma_{\mathrm{born}}
$$

where $\Pi^{\prime}(s)$ is the vacuum polarization function $\Pi^{\prime}(s) \equiv \Pi(s)-\Pi(0)$ and $\Pi(s)$ is obtained from a dispersion relation integrating over hadronic data.

\section{Acknowledgments}

We thank Miriam Fritsch and Achim Denig for discussions about experimental aspects of $e^{+} e^{-}$data, in particular those of Ref. [31. SE acknowledges the kind hospitality at the University of Mainz, and partial support by RFBR grants 10-02-695, 11-02-112, and 11-02-558.

\section{References}

[1] M.A. Shifman, A.I. Vainshtein, and V. I. Zakharov, Nucl. Phys. B 147, 385 (1979); B 147, 448 (1979).

[2] For a recent review see, e.g., P. Colangelo, and A. Khodjamirian, in: "At the Frontier of Particle Physics / Handbook of QCD", M. Shifman, ed. (World Scientific, Singapore 2001), Vol. 3, 1495.

[3] K. G. Chetyrkin, S. Narison, and V. I. Zakharov, Nucl. Phys. B550, 353 (1999).

[4] T. Lee, Phys. Rev. D82, 114021 (2010).

[5] C. A. Dominguez, and K. Schilcher, Phys. Rev. D61, 114020 (2000).

[6] C. A. Dominguez, and K. Schilcher, JHEP 0701 (2007) 093.

[7] C. A. Dominguez, N. F. Nasrallah, and K. Schilcher, Phys. Rev. D80 , 054014 (2009).

[8] A. Almasy, K. Schilcher, and H. Spiesberger, Eur. Phys. J. C55, 237 (2008).

[9] ALEPH Collaboration, S. Schael et al., Phys. Rept. 421, 191 (2005).

[10] S. I. Eidelman, L. M. Kurdadze, and A. I. Vainshtein, Phys. Lett. B82, 278 (1979); R. A. Bertlmann et al., Z. Phys. C39, 231 (1988).

[11] M. Davier et al., Eur. Phys. J. C71, 1515 (2011). 
[12] For recent reviews see, e.g., F. Jegerlehner, and A. Nyffeler, Phys. Rept. 477, 1 (2009).

[13] R. Alemany, M. Davier, and A. Hocker, Eur. Phys. J. C2, 123 (1998).

[14] F. Jegerlehner, and R. Szafron, Eur. Phys. J. C71 , 1632 (2011).

[15] S. G. Gorishnii, A. L. Kataev, and S. A. Larin, Phys. Lett. B259, 144 (1991).

[16] L. R. Surguladze, and M. A. Samuel, Phys. Rev. Lett. 66, 560 (1991).

[17] K. G. Chetyrkin, A. L. Kataev, and F. V. Tkachov, Phys. Lett. B85, 277 (1979).

[18] M. Dine, and J. R. Sapirstein, Phys. Rev. Lett. 43, 668 (1979).

[19] W. Celmaster, and R. J. Gonsalves, Phys. Rev. Lett. 44, 560 (1980).

[20] P. A. Baikov, K. Chetyrkin, and J. H. Kühn, Phys. Rev. Lett. 101, 012002 (2008).

[21] K. G. Chetyrkin, B. A. Kniehl, and M. Steinhauser, Phys. Rev. Lett. 79, 2184 (1997).

[22] G. Launer, Z. Phys. C32, 557 (1986).

[23] A. Pich, arXiv:1107.1123. For a recent review see e.g. S. Bethke et al., arXiv: 1110.0016.

[24] R. R. Akhmetshin et al., Phys. Lett. B605, 26 (2005).

[25] B. Aubert et al., Phys. Rev. Lett. 193, 231801 (2009).

[26] B. Aubert et al., Phys. Rev. D70, 072004 (2004).

[27] R. R. Akhmetshin et al., Phys. Lett. B642, 203 (2006).

[28] M. N. Achasov et al., Phys. Rev. D66, 032001 (2002).

[29] M. N. Achasov et al., Phys. Rev. D68, 052006 (2003).

[30] B. Aubert et al., Phys. Rev. D71, 052001 (2005).

[31] V. P. Druzhinin, Proc. 23rd Int. Symposium on Lepton-Photon Interactions at High Energy (LP07), Daegu, Korea, 13-18 Aug 2007, published in Daegu 2007, Lepton and Photon Interactions at High Energies 134. arXiv:0710.3455 [hep-ex]].

[32] M. N. Achasov et al., J. High Energy Phys. 109, 379 (2009).

[33] B. Aubert et al., Phys. Rev. D76, 092005 (2007).

[34] B. Aubert et al., Phys. Rev. D73, 052003 (2006).

[35] R. R. Akhmetshin et al., Phys. Lett. B562, 173 (2003).

[36] R. R. Akhmetshin et al., Phys. Lett. B669, 217 (2008).

[37] M. N. Achasov et al., Phys. Rev. D76, 072012 (2007). 
[38] D. Bisello et al., Z. Phys. C21, 13 (1988).

[39] M. N. Achasov et al., Phys. Rev. D63, 072002 (2001).

[40] M. N. Achasov et al., J. High Energy Phys. 103, 720 (2006).

[41] F. Mane et al., Nucl. Phys. B99, 261 (1981).

[42] B. Aubert et al., Phys. Rev. D77, 092002 (2008).

[43] B. Aubert et al., Phys. Rev. D73, 012005 (2006).

[44] A. Antonelli et al., Nucl. Phys. B517, 3 (1998).

[45] J. Z. Bai et al., Phys. Rev. Lett. 88, 101802 (2002).

[46] M. Ablikim et al., Phys. Lett. B677, 239 (2009).

[47] M. Davier et al., Eur. Phys. J. C27, 497 (2003).

[48] R. R. Akhmetshin et al., Phys. Lett. B578, 285 (2004).

[49] B. Aubert et al., Phys. Rev. D76, 012008 (2007).

[50] K. Hagiwara, A. D. Martin, D. Nomura, T. Teubner, Phys. Rev. D69, 093003 (2004).

[51] F. Mane et al., Thesis, Université de Paris-Sud, Report LAL 82/46, (1982).

[52] L. J. Reinders, H. Rubinstein, and S. Yazaki, Phys. Rep. C127, 1 (1985).

[53] E. Di Salvo, and M. Pallavicini, Nucl. Phys. B 427, 22 (1994).

[54] B. V. Geshkenbein, Phys. Rev. D 70, 074027 (2004). 